\title{
Poroelastic Measurement Schemes Resulting in Complete Data Sets for Granular and Other Anisotropic Porous Media
}

\author{
James G. Berryman ${ }^{1, *}$ \\ ${ }^{1}$ University of California, Lawrence Berkeley National Laboratory, \\ One Cyclotron Road MS 90R1116, Berkeley, CA 94720, USA
}

\begin{abstract}
Poroelastic analysis usually progresses from assumed knowledge of dry or drained porous media to the predicted behavior of fluid-saturated and undrained porous media. Unfortunately, the experimental situation is often incompatible with these assumptions, especially when field data (from hydrological or oil/gas reservoirs) are involved. The present work considers several different experimental scenarios typified by one in which a set of undrained poroelastic (stiffness) constants has been measured using either ultrasound or seismic wave analysis, while some or all of the dry or drained constants are normally unknown. Drained constants for such a poroelastic system can be deduced for isotropic systems from available data if a complete set of undrained compliance data for the principal stresses are available - together with a few other commonly measured quantities such as porosity, fluid bulk modulus, and grain bulk modulus. Similar results are also developed here for anisotropic systems having up to orthotropic symmetry if the system is granular (i.e., composed of solid grains assembled into a solid matrix, either by a cementation process or by applied stress) and the grains are known to be elastically homogeneous. Finally, the analysis is also fully developed for anisotropic systems with nonhomogeneous (more than one mineral type), but still isotropic, grains - as well as for uniform collections of anisotropic grains as long as their axes of symmetry are either perfectly aligned or perfectly random.
\end{abstract}

* JGBerryman@LBL . GOV 


\section{Introduction}

Poroelastic analysis [1-8] usually progresses from assumed knowledge of dry or drained porous media to the predicted behavior of fluid-saturated and undrained porous media. Unfortunately, the experimental situation is often incompatible with these assumptions, especially when field data (say from oil/gas or hydrological reservoirs) are involved.

The present work considers several different experimental scenarios typified by one in which a set of undrained constants has been measured using either ultrasound (in the laboratory) or seismic wave analysis (for field data), while some or all of the dry or drained constants are usually unmeasured and therefore unknown. Drained constants for such a poroelastic system can be deduced from available data. This goal can be achieved if a complete set of undrained compliance data (as could be calculated by inverting the stiffness data, which are almost directly obtained - within a factor of the density - from wave speed measurements) are available, together with a few other commonly measured quantities such as porosity, fluid bulk modulus, and grain bulk modulus for isotropic systems. Similar results are developed here for anisotropic systems having up to orthotropic symmetry if the system is granular (i.e., composed of solid grains assembled into solid either by a cementation process or by applied stress) and the grains are known to be elastically homogeneous.

In the later sections, the analysis is also fully developed for anisotropic systems with inhomogeneous (meaning more than one mineral type is present), but still isotropic, grains. Also studied is the case for uniform collections of the same types of anisotropic grains, as long as the grain symmetry axes are either perfectly aligned or perfectly random. We show how many poroelastic data are needed in order to consider the data sets complete, and which types of data are in some sense redundant. Some combinations can be used to replace other data types that remain missing when lab experimental and/or field limitations prevent direct measurements of all the poroelastic coefficients. 


\section{Isotropic Poroelasticity}

\subsection{Homogeneous grains}

Gassmann's [1] famous equation is sometimes written in the form

$$
K^{u}=K^{d}+\alpha^{2} /\left[(\alpha-\phi) / K^{g}+\phi / K_{f}\right]
$$

for isotropic systems, where $\alpha \equiv 1-K^{d} / K^{g}$ is the effective stress coefficient or Biot-Willis coefficient [2], $K^{g}$ is the solid modulus of the grains (assumed homogeneous), $K_{f}$ is the pore fluid modulus, and $\phi$ is the porosity. The formula becomes more complicated if the solids constituting the porous medium are heterogeneous. But we will delay discussion of this point to the next subsection and for now assume that the solids are truly homogeneous. For notational convenience, we next introduce a modulus for a fluid suspension having the same solid and fluid components as well as the same porosity, but having drained modulus $K^{d} \equiv 0$. Then we find that the effective modulus is given by

$$
K_{\text {susp }}=\left[\frac{1-\phi}{K^{g}}+\frac{\phi}{K_{f}}\right]^{-1} .
$$

In fact this result follows directly from Gassmann's formula (1) by setting $K^{d}=0$ everywhere, since then $K^{u}=K_{\text {susp }}$. But of course this result is also well-known in mechanics and acoustics (Wood [8]) for these types of fluid-solid suspensions.

\subsection{Deducing drained moduli from undrained: Isotropic system with homogeneous} grains

Rewriting Gassmann's formula in these terms, we find first that

$$
K^{u}=K^{d}+\frac{\left(1-K^{d} / K^{g}\right)^{2}}{1 / K_{\text {susp }}-K^{d} /\left(K^{g}\right)^{2}} .
$$

Note first that all explicit porosity $\phi$ dependence is now imbedded in the modulus $K_{\text {susp }}$. So if we simply multiply through by the denominator on the right hand side, we find

$$
K^{u}\left(\frac{1}{K_{\text {susp }}}-\frac{K^{d}}{\left(K^{g}\right)^{2}}\right)=1-2 \frac{K^{d}}{K^{g}}+\frac{K^{d}}{K_{\text {susp }}} .
$$

Note next that two terms of the form $\left(K^{d} / K^{g}\right)^{2}$ have cancelled from this expression. Once these convenient cancellations have occurred, $K^{d}$ appears only linearly in the resulting expression. The equation can therefore be solved immediately for drained modulus $K^{d}$ in terms 
of the undrained modulus $K^{u}$ and the other factors that are also assumed to be known (and in fact these other factors are usually easier to measure than either $K^{u}$ or $K^{d}$ ). Finally, we obtain:

$$
K^{d}=\left(\frac{K^{u}}{K_{\text {susp }}}-1\right)\left[1 / K_{\text {susp }}-2 / K^{g}+K^{u} /\left(K^{g}\right)^{2}\right]^{-1} .
$$

This result shows that the drained modulus can be deduced from measurements of the undrained modulus, together with knowledge of $\phi, K_{f}$, and $K^{g}$. Note that this result was first derived by Zhu and McMechan [10] (although not written in this form), but apparently published only in a conference proceedings.

While this isotropic result may seem elementary to some readers, and although it has also been known for some time in principle [10] (though not widely disseminated in the poroelasticity community), it is important nevertheless to point out here that this isotropic result can be generalized for anisotropic systems having at least up to orthotropic symmetry - as will be shown explicitly later in this paper. This result for anisotropic systems is one key to unraveling the drained response from the undrained response for these more complicated systems.

Another important point about both equations (4) and (5) is that they provide special insight into the relationship between drained and undrained moduli for granular systems. In particular, if the pore fluid is a liquid and the grains in such a system are only lightly pressed together, it can easily happen that $K^{g}>K^{u}>K_{\text {susp }}>>K^{d}$. When this situation holds, it is easy to show that (3), (4), and (5) all reduce approximately to

$$
K^{u} \simeq K_{\text {susp }}+K^{d}
$$

where $K^{d}$ has a very small contribution to the undrained modulus compared to that of the suspension modulus. This result turns out to be quite important for analysis of the behavior of liquid-saturated granular materials at lower confining stresses $(<5 \mathrm{MPa})$.

Also note that the approximate form (6) holds as well in the opposite extreme in which $K_{\text {susp }}$ is very small - for example when the system is air or gas saturated - while the drained value $K^{d}$ is not small - as could occur in granular media under high confining stress.

In either of these situations, a good approximation to $K^{d}$ is found by taking the difference of $K^{u}$ and $K_{\text {susp }}$. 


\subsection{Heterogeneous grains}

When the grains in a granular packing are NOT composed of elastically homogeneous and isotropic materials, or if they are homogeneous but anisotropic while nevertheless being distributed in a randomly oriented way in space, then - as has been pointed out previously by Brown and Korringa [3], Rice and Cleary [4], and others [6-8] - we need to introduce a more general notation to deal with these circumstances.

Recall that the Reuss average [11] of the grain bulk moduli when a distribution of grain types is present is then given by:

$$
\frac{1}{K_{R}^{g}} \equiv \sum_{m=1, \ldots, n} \frac{v_{m}}{K_{m}}
$$

where $v_{m}$ is the volume fraction (out of all the solid material present, so that $\sum_{m} v_{m}=1$ ) of the $m$-th isotropic grain having bulk modulus $K_{m}$. This average should to be distinguished from that of the Voigt [12] average

$$
K_{V}^{g} \equiv \sum_{m=1, \ldots, n} v_{m} K_{m}
$$

which is known [13] to satisfy $K_{V}^{g} \geq K_{R}^{g}$, and furthermore these two measures are also known [11-13] to satisfy $K_{V}^{g} \geq K_{g}^{*} \geq K_{R}^{g}$, where $K_{g}^{*}$ is the effective bulk modulus of an isotropic elastic composite consisting only of the minerals $m=1, \ldots, n$ in the same volume proportions given by the $v_{m}$ values. However, this fact actually is not pertinent here as the only averages of this type that play a direct role in the poroelastic equations are always those of the Reuss-type, as we shall see in further developments.

To clarify our later usage of the same notation $K_{R}^{g}$, we emphasize here that when (or if) the grains in our assemblage are all anisotropic - but nevertheless of the same type and oriented randomly in space - then the pertinent average is again the Reuss average. But in this case the average is determined by the equation

$$
\frac{1}{K_{R}^{g}}=\sum_{i, j=1,2,3} s_{i j}^{g},
$$

where the $s_{i j}^{g}$ for $i, j=1,2,3$ are the principal components of the compliance matrix for the anisotropic grain material itself. It is easy to see that this must be the case if we refer back to the equations above, specifically those requiring the suspension result $K_{\text {susp }}$. The formula 
as quoted in (2) was only written for the case of homogeneous grains. But if we generalize this formula slightly as:

$$
K_{\text {susp }} \equiv\left[\frac{1-\phi}{K_{R}^{g}}+\frac{\phi}{K_{f}}\right]^{-1},
$$

then we see that it holds equally true: (a) for homogeneous isotropic grains (when $K_{R}^{g} \equiv K^{g}$ ),

(b) for an heterogeneous volume of isotropic grains [when $K_{R}^{g}$ is given by (7)], or (c) for anisotropic grains when they are randomly oriented in the fluid [and then $K_{R}^{g}$ is given by (9)]. In all these cases, we do assume that this mixture of grains and fluid is close to being a true suspension, by which we mean that individual grains are acted on similarly by changes in fluid pressure. If the clumpings of granular materials are loose enough, then the fluid can act equally on all the individual grains, and the result in (10) holds true regardless of the heterogeneity. However, if this is not the case, then there must be elastically distinct clumpings of grains forming solid composites locally - so the individual grains are no longer uniformly surrounded by the pore fluid - then each grain's fluid environment is different, due to welded contacts with other contiguous grains. We are assuming for the present purposes that such effects are negligible in the types of comparatively homogeneous granular porous media (homogeneous on the meso- and macroscales, but not necessarily on the microscale) being studied here. In fact, some types of more heterogeneous systems can be treated and some of these have already been studied [14,15] when the porous system is composed of just two distinct types of grain clumpings; however, we will not be studying such double-porosity and/or multi-porosity effects in the present paper.

\subsection{Heterogeneous pores}

Another important type of heterogeneity that can occur in practice involves heterogeneity of the pore space [3]. One obvious issue is whether the pores are all connected to each other, or whether there may be two (or more) distinct, but intertwining, pore systems. One wellknown example of this situation is the double-porosity concept [14-17], in which one type of pore has high volume but low permeability, while the other has low volume (imagine a system of very flat cracks or fractures) and high permeability. We can also consider that some pores might be interior to some grains and not connected to any other pores (and might therefore also be empty of pore fluid), while other subsets of the grains have no inherent porosity of this type, and so are truly solid grains. 
We will not try to deal with all these cases simultaneously, as even enumerating all the possibilities quickly becomes burdensome. We will limit ourselves instead to one of the more typical scenarios considered for example by Brown and Korringa [3] and by Rice and Cleary [4] — and also see the recent related work of Gurevich et al. [18].

Heterogeneity of the pore space is most important when we consider flow of fluid into and out of the boundaries of our study sample. Then, the concept of increment of fluid content $\zeta$ comes into play, and special care is required. A straightforward definition of this dimensionless parameter (just as the strains $e_{11}, e_{22}, \ldots, e_{13}$ are all dimensionless) is given by:

$$
\zeta \equiv \frac{\delta(\phi V)-\delta V_{f}}{V} \simeq \phi\left(\frac{\delta V_{\phi}}{V_{\phi}}-\frac{\delta V_{f}}{V_{f}}\right),
$$

where $V$ is the overall volume of the initially fully fluid-saturated porous material at the first instant of consideration, $V_{\phi}=\phi V$ is the pore volume, with $\phi$ being the fluid-saturated porosity of the volume, $V_{f}$ is the volume occupied by the pore-fluid, and so $V_{f}=\phi V$ initially. The $\delta$ 's indicate small changes in the quantities immediately following them. For "drained" systems, there must be a reservoir of the same fluid just outside the volume $V$ that can either supply more fluid or absorb any excreted fluid as needed during the nonstationary phase of the poroelastic process; the amount of pore fluid can therefore either increase or decrease from the initial amount of pore fluid, and at the same time the pore volume can also be changing, but not necessarily at exactly the same rate as the pore fluid itself. The one exception to these statements is when the surface pores of the total volume $V$ are sealed, in which case the system is "undrained" and $\zeta \equiv 0$, identically. In these circumstances, it is still possible that $V_{f}$ and $V_{\phi}=\phi V$ are both changing, but because of the imposed undrained boundary conditions, they are necessarily changing at the same rate. The result is that, for an isotropic system, we have:

$$
\zeta=\phi\left[\frac{\delta \sigma_{c}}{K_{p}}+\delta p_{f}\left(\frac{1}{K_{p}}-\frac{1}{K_{R}^{\phi}}+\frac{1}{K_{f}}\right)\right],
$$

where the various moduli in (12) are defined by the following relations (also see [3]):

$$
\begin{gathered}
-\frac{\delta V_{f}}{V_{f}}=\frac{\delta p_{f}}{K_{f}} \\
-\frac{\delta V}{V}=\frac{\delta p_{d}}{K_{R}^{d}}+\frac{\delta p_{f}}{K_{R}^{g}},
\end{gathered}
$$


and

$$
-\frac{\delta V_{\phi}}{V_{\phi}}=\frac{\delta p_{d}}{K_{p}}+\frac{\delta p_{f}}{K_{R}^{\phi}} .
$$

The changes in fluid pressure and differential pressure are respectively $\delta p_{f}$ and $\delta p_{d} \equiv \delta p_{c}-$ $\delta p_{f}$, where $\delta p_{c}=-\delta \sigma_{c}$ is the uniform confining pressure, if the external confining pressure is uniform. If not, then this quantity is replaced in the definition of $\delta p_{c}$ by $-\delta \sigma_{m}$, which is the change in the mean confining pressure and where $\sigma_{m} \equiv\left(\sigma_{11}+\sigma_{22}+\sigma_{33}\right) / 3$ is the definition of the mean principal stress. Clearly, if the confining principal stress is uniform $\left(\sigma_{11}=\sigma_{22}=\sigma_{33}\right)$, then the mean stress equals this uniform confining stress. If not, then there can be additional shearing effects that need to be taken into account, but these do not play any role in the changes of fluid content since this quantity is effectively a measure only of the total number of fluid particles contained in the pertinent pore volume.

It can also be shown using poroelastic reciprocity (and we will see this later as it very clearly develops in the following anisotropic analysis) that

$$
\frac{\phi}{K_{p}}=\frac{\alpha_{R}}{K_{R}^{d}}=\frac{1}{K_{R}^{d}}-\frac{1}{K_{R}^{g}}
$$

Then we can generalize Gassmann's formula for undrained modulus so it takes the form:

$$
\frac{1}{K_{R}^{u}}=\frac{1-\alpha_{R} B}{K_{R}^{d}}
$$

where $\alpha_{R}=1-K_{R}^{d} / K_{R}^{g}$ and

$$
B=\left(\frac{1}{K_{R}^{d}}-\frac{1}{K_{R}^{g}}\right)\left[\left(\frac{1}{K_{R}^{d}}-\frac{1}{K_{R}^{g}}\right)+\phi\left(\frac{1}{K_{f}}-\frac{1}{K_{R}^{\phi}}\right)\right]^{-1}
$$

is Skempton's $[19,20]$ second coefficient. Combining these terms, we find that the most general form of the equation for the undrained bulk modulus in the isotropic case is:

$$
\frac{1}{K_{R}^{u}}=\frac{1}{K_{R}^{d}}-\left(\frac{1}{K_{R}^{d}}-\frac{1}{K_{R}^{g}}\right)^{2}\left[\left(\frac{1}{K_{R}^{d}}-\frac{1}{K_{R}^{g}}\right)+\phi\left(\frac{1}{K_{f}}-\frac{1}{K_{R}^{\phi}}\right)\right]^{-1},
$$

or, alternatively,

$$
\frac{1}{K_{R}^{u}}=\frac{1}{K_{R}^{d}}-\frac{\left(\alpha_{R} / K_{R}^{d}\right)^{2}}{\alpha_{R} / K_{R}^{d}+\phi\left(\frac{1}{K_{f}}-\frac{1}{K_{R}^{\phi}}\right)},
$$

which is the isotropic result of Brown and Korringa [3], and should also be compared directly to (1). We see that, if the pore modulus and grain modulus are equal, so $K_{R}^{\phi}=K_{R}^{g}$, then (20) reduces exactly to (1). Although this result is the same as that of Brown and Korringa [3], we nevertheless write it differently to emphasize different features. 


\subsection{Deducing drained constants from undrained: Heterogeneous grains and pores}

We were able to deduce $K_{R}^{d}$ from our knowledge of $K_{R}^{u}, K_{R}^{g}, K_{f}$, and $\phi$ in subsection 2.2 . But even though we are still assuming the system is isotropic, we have now introduced some additional degrees of freedom by permitting the grains and pores to be heterogeneous. It is clear that we cannot deduce $K_{R}^{d}$ if we have only the same amount of information as before.

In particular, it does seem fairly straightforward to measure $K_{R}^{g}$, since we have already described its meaning in the earlier discussion and even given formulas for it — while requiring information about the constituents and their volume fractions, or alternatively about the principal components of elastic compliance and/or stiffness matrices. But we have another variable now, which is the pore modulus $K_{R}^{\phi}$, and this bulk modulus is not so easy either to model or to measure directly [21]. However, if we add one more piece of information a fact that should typically be known in poroelastic systems - namely the second Skempton coefficient $B=p_{f} / p_{c}$, then it turns out that we can solve for both $K_{R}^{d}$ and $K_{R}^{\phi}$. So again we do assume that $K_{R}^{g}$ and $K_{R}^{u}$ are known. But now we assume that $B$ is also known experimentally. Working through the algebra, we find that

$$
K_{R}^{d}=\frac{1-B}{1 / K_{R}^{u}-B / K_{R}^{g}}
$$

[which is a rearrangement of $\left.K_{R}^{u}=K_{R}^{d} /\left(1-\alpha_{R} B\right)\right]$, and similarly that

$$
\frac{1}{K_{R}^{\phi}}=\frac{1}{K_{f}}-\left(\frac{1-B}{\phi B}\right)\left(\frac{1}{K_{R}^{d}}-\frac{1}{K_{R}^{g}}\right)=\frac{1}{K_{f}}-\left(\frac{1}{\phi B}\right)\left(\frac{1}{K_{R}^{u}}-\frac{1}{K_{R}^{g}}\right) .
$$

In (22), we used the previous result (21) for $K_{R}^{d}$ to simplify the final formula.

These forms are very useful for our applications in poroelasticity, but so far they apply only to the fully isotropic case. We next show that a very similar set of formulas applies to the anisotropic cases under consideration. We also gain greater clarity at this point by switching to the more general anisotropic problem, where we can see more easily how poroelastic reciprocity comes directly into play.

\section{Anisotropic Poroelasticity}

If the overall porous medium is anisotropic either due to some preferential alignment of the constituent particles or due to externally imposed stress (such as a gravity field and 
weight of overburden, for example), we consider the orthorhombic anisotropic version of the poroelastic equations:

$$
\left(\begin{array}{c}
e_{11} \\
e_{22} \\
e_{33} \\
-\zeta
\end{array}\right)=\left(\begin{array}{cccc}
s_{11} & s_{12} & s_{13} & -\beta_{1} \\
s_{12} & s_{22} & s_{23} & -\beta_{2} \\
s_{13} & s_{23} & s_{33} & -\beta_{3} \\
-\beta_{1} & -\beta_{2} & -\beta_{3} & \gamma
\end{array}\right)\left(\begin{array}{c}
\sigma_{11} \\
\sigma_{22} \\
\sigma_{33} \\
-p_{f}
\end{array}\right) .
$$

From here on throughout the paper, we drop the $\delta$ 's from the stresses and strains, as this extra notation is truly redundant when they are all being treated as small (and therefore resulting in linear effects) as we do here, for small deviations from an initial rest state.

The $e_{i i}$ (no summation over repeated indices) are strains in the $i=1,2,3$ directions. The $\sigma_{i i}$ are the corresponding stresses. The fluid pressure is $p_{f}$. The increment of fluid content is $\zeta$. The drained compliances are $s_{i j}=s_{i j}^{d}$. Undrained compliances (not yet shown) are symbolized by $s_{i j}^{u}$. Coefficients $\beta_{i}=s_{i 1}+s_{i 2}+s_{i 3}-1 / 3 K_{R}^{g}$, where $K_{R}^{g}$ is again the Reuss average modulus of the grains. The drained Reuss average bulk modulus is defined by

$$
\frac{1}{K_{R}^{d}}=\sum_{i j=1,2,3} s_{i j}^{d} .
$$

For the Reuss average undrained bulk modulus $K_{R}^{u}$, we have drained compliances replaced by undrained compliances. A similar definition $(9)$ of $K_{R}^{g}$, with drained compliances replaced by grain compliances has already been introduced earlier in our discussion. The alternative Voigt [12] average (also see [13]) of the stiffnesses will play no role in the present work. And, finally, $\gamma=\sum_{i=1-3} \beta_{i} / B K_{R}^{d}$, where $B$ is the second Skempton coefficient of [19], which will be defined carefully again later in our discussion.

The shear terms due to twisting motions (i.e., strains $e_{23}, e_{31}, e_{12}$ and stresses $\sigma_{23}, \sigma_{31}$, $\left.\sigma_{12}\right)$ are excluded from this discussion since they typically do not couple to the modes of interest for anisotropic systems having orthotropic symmetry, or any more symmetric system such as those being either transversely isotropic or isotropic. We have also assumed that we know the true axes of symmetry, and make use of them in our formulation of the problem. Note that the $s_{i j}$ 's are the elements of the compliance matrix $\mathbf{S}$ and are all independent of the fluid, and therefore would be the same if the medium were treated as elastic (i.e., by ignoring the fluid pressure, or assuming that the fluid saturant is air - or vacuum). In keeping with the earlier discussions, we typically call these compliances 
the drained compliances and the corresponding matrix the drained compliance matrix $\mathbf{S}^{d}$, since the fluids do not contribute to the stored mechanical energy if they are free to drain into a surrounding reservoir containing the same type of fluid. In contrast, the undrained compliance matrix $\mathbf{S}^{u}$ presupposes that the fluid is trapped (unable to drain from the system into an adjacent reservoir) and therefore contributes in a significant and measurable way to the compliance and stiffness $\left(\mathbf{C}^{u}=\left[\mathbf{S}^{u}\right]^{-1}\right)$, and also therefore to the stored mechanical energy of the undrained system.

Although the significance of the formula is somewhat different now, we find again that

$$
\beta_{1}+\beta_{2}+\beta_{3}=\frac{1}{K_{R}^{d}}-\frac{1}{K_{R}^{g}}=\frac{\alpha_{R}}{K_{R}^{d}}
$$

if we also define (as we did for the isotropic case) a Reuss effective stress coefficient:

$$
\alpha_{R} \equiv 1-K_{R}^{d} / K_{R}^{g}
$$

Furthermore, we have

$$
\gamma=\frac{\beta_{1}+\beta_{2}+\beta_{3}}{B}=\frac{\alpha_{R}}{K_{R}^{d}}+\phi\left(\frac{1}{K_{f}}-\frac{1}{K_{R}^{\phi}}\right),
$$

since we have the rigorous result in this notation [22] that Skempton's $B$ coefficient is given by

$$
B \equiv \frac{1-K_{R}^{d} / K_{R}^{u}}{1-K_{R}^{d} / K_{R}^{g}}=\frac{\alpha_{R} / K_{R}^{d}}{\alpha_{R} / K_{R}^{d}+\phi\left(1 / K_{f}-1 / K_{R}^{\phi}\right)} .
$$

Note that both (27) and (28) contain dependence on the pore bulk modulus $K_{R}^{\phi}$ that comes into play when the pores are heterogeneous, regardless of whether the system is isotropic or anisotropic. We emphasize that all these formulas are rigorous statements based on the earlier anisotropic analysis. The appearance of $K_{R}^{d}$ and $\alpha_{R}$ is not an approximation, but merely a useful choice of notation made here because it will enable us to see clearly the similarity between the rigorous anisotropic formulas and the isotropic ones.

\subsection{The $\beta_{i}$ coefficients}

We will now provide several results for the $\beta_{i}$ coefficients, and then follow the results with a general proof of their correctness. 
In many important and useful cases, the coefficients $\beta_{i}$ are determined by

$$
\beta_{i}=s_{i 1}^{d}+s_{i 2}^{d}+s_{i 3}^{d}-\frac{1}{3 K_{R}^{g}} .
$$

Again, $K_{R}^{g}$ is the Reuss average of the grain modulus, since the local grain modulus is not necessarily assumed uniform here as discussed previously. Equation (29) holds true for homogeneous grains, such that $K_{R}^{g}=K^{g}$. It also holds true for the case when $K_{R}^{g}$ is determined instead by (7). However, when the grains themselves are anisotropic, we need to allow again for this possibility, and this can be accomplished by defining three directional grain bulk moduli determined by:

$$
\frac{1}{3 \bar{K}_{i}^{g}} \equiv s_{i 1}^{g}+s_{i 2}^{g}+s_{i 3}^{g}=s_{1 i}^{g}+s_{2 i}^{g}+s_{3 i}^{g}
$$

for $i=1,2,3$. The second equality follows because the compliance matrix is always symmetric. We call these quantities in (30) the partial grain-compliance sums, and the $\bar{K}_{i}^{g}$ are the directional grain bulk moduli. Then, the formula for (29) is replaced by

$$
\beta_{i}=s_{i 1}^{d}+s_{i 2}^{d}+s_{i 3}^{d}-\frac{1}{3 \bar{K}_{i}^{g}}
$$

Note that the factors of three have been correctly accounted for because

$$
\sum_{i=1,2,3} \frac{1}{3 \bar{K}_{i}^{g}}=\frac{1}{K_{R}^{g}}
$$

in agreement with (9). If the three contributions represented by (30) for $i=1,2,3$ happen to be equal, then clearly each equals one-third of the sum (32).

The preceding results are for perfectly aligned grains. If the grains are instead perfectly randomly oriented, then it is clear that the formulas in (29) hold as before, but now $K_{R}^{g}$ is determined instead by (9).

All of these statements about the $\beta_{i}$ are easily proven by considering the situation when $\sigma_{11}=\sigma_{22}=\sigma_{33}=-p_{c}=-p_{f}$. Because then, from (23), we have:

$$
-e_{i i}=\left(s_{i 1}^{d}+s_{i 2}^{d}+s_{i 3}^{d}\right) p_{c}+\beta_{i}\left(-p_{f}\right)=\left(s_{i 1}^{g}+s_{i 2}^{g}+s_{i 3}^{g}\right) p_{f} \equiv \frac{p_{f}}{3 \bar{K}_{i}^{g}}
$$

in the most general of the three cases discussed, and holding true for each value of $i=1,2,3$. This is a statement about the strain $e_{i i}$ that would be observed in this situation, as it must be the same if these anisotropic (or inhomogeneous) grains were immersed in the fluid, while 
measurements were taken of the strains observed in each of the three directions $i=1,2,3$, during variations of the fluid pressure $p_{f}$. We may consider this proof to be a thought experiment for determining the coefficients, in the same spirit as those proposed originally by Biot and Willis [2,23] for the isotropic and homogeneous case.

\subsection{Coefficient $\gamma$}

The relationship of coefficient $\gamma$ to the other coefficients is easily established because we have already discussed the main issue, which involves determining the role of the various other constants contained in Skempton's coefficient $B$ [19]. We have quoted this result in (18).

Again, from (23), we find that

$$
-\zeta=0=-\left(\beta_{1}+\beta_{2}+\beta_{3}\right) \sigma_{c}-\gamma p_{f}
$$

for undrained boundary conditions. Thus, we find again that

$$
\frac{p_{f}}{p_{c}} \equiv B=\frac{\beta_{1}+\beta_{2}+\beta_{3}}{\gamma},
$$

where $p_{c}=-\sigma_{c}$ is the confining pressure. Thus, the scalar coefficient $\gamma$ is determined immediately and given by

$$
\gamma=\frac{\beta_{1}+\beta_{2}+\beta_{3}}{B}=\frac{\alpha_{R} / K_{R}^{d}}{B}=\alpha_{R} / K_{R}^{d}+\phi\left(\frac{1}{K_{f}}-\frac{1}{K_{R}^{\phi}}\right) .
$$

Alternatively, we could say that

$$
B=\frac{\alpha_{R}}{\gamma K_{R}^{d}}
$$

We have now determined the physical/mechanical significance of all the coefficients in the poroelastic matrix (23). These results are as general as possible without considering poroelastic symmetries that have less than orthotropic symmetry, while also taking advantage of our assumption that we do typically know the three directions of the principal axes of symmetry.

\subsection{Inverting poroelastic compliance}

The matrix in (23) is in compliance form and has extremely simple poroelastic behavior in the sense that all the fluid mechanical effects appear only in the single coefficient $\gamma$. We 
can simplify the notation a little more by lumping some coefficients together, combining the $3 \times 3$ submatrix in the upper left corner of the matrix in (23) as $\mathbf{S}$, and defining the column vector $\mathbf{b}$ by

$$
\mathbf{b}^{T} \equiv\left(\beta_{1}, \beta_{2}, \beta_{3}\right)
$$

The resulting $4 \times 4$ matrix and its inverse are now related by:

$$
\left(\begin{array}{cc}
\mathbf{S} & -\mathbf{b} \\
-\mathbf{b}^{T} & \gamma
\end{array}\right)=\left(\begin{array}{cc}
\mathbf{A} & \mathbf{q} \\
\mathbf{q}^{T} & z
\end{array}\right)^{-1}
$$

where the elements of the inverse matrix can be shown to be written in terms of drained stiffness matrix $\mathbf{C}^{d}=\mathbf{C}=\mathbf{S}^{-1}$ by introducing three components: $(a)$ scalar $z=\left[\gamma-\mathbf{b}^{T} \mathbf{C b}\right]^{-1}$, (b) column vector $\mathbf{q}=z \mathbf{C b}$, and $(c)$ undrained $3 \times 3$ stiffness matrix (i.e., the pertinent one connecting the principal strains to principal stresses) is given by $\mathbf{A}=\mathbf{C}+z \mathbf{C b b}^{T} \mathbf{C}=$ $\mathbf{C}^{d}+z^{-1} \mathbf{q} \mathbf{q}^{T} \equiv \mathbf{C}^{u}$, since $\mathbf{C}^{d}$ is drained stiffness and $\mathbf{A}=\mathbf{C}^{u}$ is clearly undrained stiffness by construction. This result is the same as that of Gassmann [1] for anisotropic porous media, although his results were presented in a form somewhat harder to scan than the form shown here.

Also, note the important fact that the observed decoupling of the fluid effects occurs only in the compliance form (23) of the equations, and never in the stiffness (inverse) form for the poroelasticity equations.

From these results, it is not hard to show that

$$
\mathbf{S}^{d}=\mathbf{S}^{u}+\gamma^{-1} \mathbf{b b}^{T}
$$

This result emphasizes the remarkably simple fact that the drained compliance matrix can be found directly from knowledge of the inverse of undrained stiffness, and the still unknown, but sometimes relatively easy to estimate, values of $\gamma$, together with the three distinct orthotropic $\beta_{i}$ coefficients, for $i=1,2,3$. 


\subsection{Deducing coefficients from measurements: Anisotropic example with homoge-} neous grains

Now progress is made by considering the Reuss average again for both the orthotropic drained and undrained compliances:

$$
\frac{1}{K_{R}^{d}} \equiv \sum_{i, j=1,2,3} s_{i j}^{d}
$$

and

$$
\frac{1}{K_{R}^{u}} \equiv \sum_{i, j=1,2,3} s_{i j}^{u} .
$$

These effective moduli are the Reuss averages of the nine compliances in the upper left $3 \times 3$ of the full (including the uncoupled shear components) $6 \times 6$ compliance matrix for the two cases, respectivley, when the pore fluid is allowed to drain from the porous system, and when the pore fluid is trapped by a jacketing material and therefore undrained.

Although the significance of the formula in the anisotropic case is somewhat different now, we find again that

$$
\beta_{1}+\beta_{2}+\beta_{3}=\frac{1}{K_{R}^{d}}-\frac{1}{K_{R}^{g}}=\frac{\alpha_{R}}{K_{R}^{d}}
$$

if we also define a Reuss effective stress coefficient:

$$
\alpha_{R} \equiv 1-K_{R}^{d} / K_{R}^{g}
$$

by analogy to the isotropic case. Furthermore, we have

$$
\gamma=\frac{\beta_{1}+\beta_{2}+\beta_{3}}{B}=\frac{\alpha_{R}}{K_{R}^{d}}+\phi\left(\frac{1}{K_{f}}-\frac{1}{K_{R}^{g}}\right),
$$

since we have the rigorous result [22] in this notation that Skempton's $B$ coefficient [19] is given by

$$
B \equiv \frac{1-K_{R}^{d} / K_{R}^{u}}{1-K_{R}^{d} / K_{R}^{g}}=\frac{\alpha_{R} / K_{R}^{d}}{\alpha_{R} / K_{R}^{d}+\phi\left(1 / K_{f}-1 / K_{R}^{g}\right)} .
$$

We should emphasize that all these formulas are rigorous statements based on the earlier anisotropic analysis. Again, the appearance of $K_{R}^{d}$ and $\alpha_{R}$ is not an approximation. In fact it is important now (it was not important in the isotropic cases considered earlier as long as the grains were also homogeneous) to make this distinction between the Reuss and Voigt averages. This choice of notation will help us to demonstrate conceptually useful analogies between the rigorous anisotropic formulas and the isotropic ones. We have prepared the way 
for these analogies by using the Reuss averages earlier in our notation, even though they were mostly redundant in those isotropic cases.

First note that, from (43) and (45), it follows that $\gamma^{-1}=\frac{B K_{R}^{d}}{\alpha_{R}}-$ also see (37). So now we can rearrange (40) to give the formal relationship

$$
s_{i j}^{d}=s_{i j}^{u}+\frac{B K_{R}^{d}}{\alpha_{R}} \beta_{i} \beta_{j}, \quad \text { for } \quad i, j=1,2,3 .
$$

At this point in the analysis, we know everything needed except for actually determining the three coefficients $\beta_{i}$, for $i=1,2,3$. But, by taking appropriate sums of (47) and using (43), we find that

$$
\beta_{i}=s_{i 1}^{d}+s_{i 2}^{d}+s_{i 3}^{d}-\frac{1}{3 K_{R}^{g}}=s_{i 1}^{u}+s_{i 2}^{u}+s_{i 3}^{u}-\frac{1}{3 K_{R}^{g}}+B \beta_{i},
$$

Rearranging, we find that

$$
\beta_{i}(1-B)=s_{i 1}^{u}+s_{i 2}^{u}+s_{i 3}^{u}-\frac{1}{3 K_{R}^{g}} .
$$

Formula (46) for Skempton's [19] coefficient determines $B$ exactly in terms of (presumed) known quantities. In the present case, the Skempton coefficient $B$ was not assumed to be known, since for homogeneous grains we can compute $K_{R}^{d}$ relatively easily, and then $B$ follows since we also know $K_{R}^{g}$. [For the case of heterogeneous or anisotropic grains, the necessary introduction of the additional variable $K_{R}^{\phi}$ requires us to have still more measured data, and it turns out that the next easiest quantity to measure is $B$ itself - as we already saw in the isotropic case.] So, all three $\beta_{i}$ 's (which are themselves drained constants) and $\gamma$ are now

precisely determined. All the remaining drained compliances $s_{i j}^{d}$ can then be found directly from (47). Note that all the steps in this inversion procedure are linear; there was no need to solve any quadratic equation in this formulation of the undrained-to-drained inversion problem. There is also no iteration, and no fitting steps are required in this procedure.

\subsection{Deducing anisotropic drained constants from undrained: Homogeneous grains} and pores

We are now in position to develop the analogy between the isotropic and anisotropic Gassmann [1] equations for the case of homogeneous grains. In particular, the equation for the suspension modulus in (2) does not change at all. However, the equation for the 
effective undrained bulk modulus $K^{u}$, as shown in both (1) and (3), changes only in that the relationship is now between the Reuss averages $K_{R}^{u}$ and $K_{R}^{d}$ of these quantities. This result is completely analogous to (3), and so will not be shown here.

Since the remainder of the argument is virtually identical to the isotropic case, we therefore obtain:

$$
K_{R}^{d}=\left(\frac{K_{R}^{u}}{K_{\text {susp }}}-1\right)\left[1 / K_{\text {susp }}-2 / K_{R}^{g}+K_{R}^{u} /\left(K_{R}^{g}\right)^{2}\right]^{-1} .
$$

This formula shows how to invert for drained Reuss bulk modulus $K_{R}^{d}$ from knowledge of $K_{R}^{u}, \phi, K_{f}$ and $K_{R}^{g}$ in an anisotropic (up to orthotropic) poroelastic system.

Clearly this formula does not yet give us the individual compliance matrix elements $s_{i j}^{d}$ directly. Nevertheless, Equation (50) was the hardest step in the overall procedure. The rest of the steps follow easily once we have this rigorous result available for our use.

To finish our analysis, we make use of the newly computed value of $K_{R}^{d}$, and substitute this number into the formula for $B$, which in this case is:

$$
B=\frac{1-K_{R}^{d} / K_{R}^{u}}{1-K_{R}^{d} / K_{R}^{g}}
$$

Once we know Skempton coefficient $B$, this value can be substituted into (49) in order to determine the $\beta_{i}$ coefficients for $i=1,2,3$. The remaining coefficient is $\gamma=\alpha_{R} / B K_{R}^{d}$. So we see that the critical step in this process was determining the value of the drained Reuss bulk modulus $K_{R}^{d}$, as was claimed earlier.

\subsection{Deducing anisotropic drained constants from undrained: Heterogeneous grains} and pores

One difficulty for heterogeneous grains comes from the additional constant $K_{R}^{\phi}$ that we do not know how to determine independently from the other poroelastic measurements. But this fundamental problem is actually no different for the anisotropic case than it was for the isotropic one, and the solution is also the same. In both cases, we need more information, and in both cases the necessary information will most likely come from our knowledge of Skempton's coefficient $B$ [19]. If we assume that $B$ can be directly measured (which is plausible, since $B=p_{f} / p_{c}$ in the undrained case when a uniform confining pressure is applied to the system), then our problem is completely solved, because $B$ is the key to solving for the coefficients $\beta_{i}$ in (49). The only new difficulty is that the terms of the form 
$1 / 3 K_{R}^{g}$ must also be replaced by the partial grain compliance sums $\frac{1}{3 \bar{K}_{i}^{g}}$, as shown in (31). So we now have

$$
\beta_{i}=s_{i 1}^{d}+s_{i 2}^{d}+s_{i 3}^{d}-\frac{1}{3 \bar{K}_{i}^{g}}=s_{i 1}^{u}+s_{i 2}^{u}+s_{i 3}^{u}-\frac{1}{3 \bar{K}_{i}^{g}}+B \beta_{i},
$$

Rearranging, the final result for heterogeneous grains is:

$$
\beta_{i}(1-B)=s_{i 1}^{u}+s_{i 2}^{u}+s_{i 3}^{u}-\frac{1}{3 \bar{K}_{i}^{g}} .
$$

So, we are almost done now, but we still need either to determine the values of the anisotropic grain correction terms $\frac{1}{3 \bar{K}_{i}^{g}}$, or to find some way of avoiding the necessity of doing so.

In principle, this can be done experimentally by actually performing a test on the porous sample that applies the same pressure inside and outside. Then, measurements of the change in strain in the three orthogonal directions $i=1,2,3$ would provide direct measures of the quantities $\bar{K}_{i}^{g}$ that we need. So this approach is one that is experimentally feasible.

An alternative that we have not considered so far could involve laboratory shear tests by applying nonzero deviatoric stress changes [19,24]. The undrained fluid pressure is given by $p_{f}=B p_{c}=B\left(-\sigma_{m}\right)$, where the mean stress is $\sigma_{m}=\left(\sigma_{11}+\sigma_{22}+\sigma_{33}\right) / 3$. But, if the $\sigma_{i i}$ 's are not uniform, then there are also deviatoric stresses present, due to the nonuniformity of the principal stresses.

\subsubsection{Triaxial testing geometry}

One common example of this type of measurement uses triaxial testing [24], where a twosided confining stress is defined as $\sigma_{22}=\sigma_{33}$, and then the deviatoric stress is determined by

$$
\sigma_{d e v} \equiv\left(\sigma_{11}-\sigma_{33}\right) / 2
$$

In this situation, the general equation relating undrained pressure to the confining stresses is given by:

$$
-p_{f}=B \sigma_{m}+2\left(A-\frac{1}{3}\right) B \sigma_{d e v},
$$

where the only new symbol is Skempton's [19] first coefficient $A$. It is not difficult to show that, in terms of our previous definitions for the triaxial testing geometry, the coefficient $A$ is given precisely by the ratio

$$
A \equiv \frac{\beta_{1}}{\beta_{1}+\beta_{2}+\beta_{3}}
$$


For an isotropic system, $A=1 / 3$, and so this contribution always vanishes in (55). This fact explains why we did not encounter this coefficient before in our analysis. Note that there is no assumption here that the poroelastic system itself is necessarily transversely isotropic. Only the prescribed equality of the two applied transverse stresses, $\sigma_{22}$ and $\sigma_{33}$, is assumed. Then, the formula (55) follows directly from the equations already presented.

\subsubsection{Non-triaxial testing geometries}

Clearly, it would also be natural to introduce other measures of the $\beta_{i}$ coefficients as well, especially if the measurements are not being constrained to the triaxial testing configuration. So we could imagine that three such coefficients might be measured according to:

$$
-p_{f}=B\left[\sigma_{m}+\sum_{i} A_{i}\left(\sigma_{i i}-\sigma_{m}\right)\right] \text {, }
$$

where

$$
A_{i}=\frac{\beta_{i}}{\beta_{1}+\beta_{2}+\beta_{3}}
$$

for $i=1,2,3$. In general, no more than two of these $A_{i}$ coefficients can be independent since $\sum_{i=1,2,3} A_{i} \equiv 1$. But, for general testing configurations, there could be two useful and distinct measurements to be gathered from deviatoric response testing, although only one was available in the triaxial testing configuration.

To be able to deduce the values of the $\beta_{i}$ 's from the $A_{i}$ 's, we need to know the value of the sum $\beta_{1}+\beta_{2}+\beta_{3}=\gamma B$. We also need to know the value of $B$ to determine any of the $A_{i}$ 's, but the value of $\gamma$ is harder to determine independently. The values of $K_{R}^{u}$ and the

total $K_{R}^{g}$ are both usually easier to determine, so it is likely we have enough information to compute the $\beta_{i}$ sum this way:

$$
\beta_{1}+\beta_{2}+\beta_{3}=\frac{\frac{1}{K_{R}^{u}}-\frac{1}{K_{R}^{g}}}{1-B}=\gamma B .
$$

If the $\beta_{i}$ sum has been computed using (58) and (59), then clearly we also have

$$
\beta_{i}=A_{i} \gamma B=A_{i}\left(\frac{\frac{1}{K_{R}^{u}}-\frac{1}{K_{R}^{g}}}{1-B}\right) .
$$

Once we have computed the $\beta_{i}$ 's, then we can also find (if we want to, although it is not usually critical information) the values of the partial sums of the grain Reuss modulus:

$$
\frac{1}{3 \bar{K}_{i}^{g}} \equiv s_{i 1}^{u}+s_{i 2}^{u}+s_{i 3}^{u}-\beta_{i}(1-B) .
$$


This additional information may therefore be available if needed for some other reason such as determining some useful descriptive information such as how well-stirred (i.e., how evenly distributed in space) the particles composing a given granular medium might be.

\subsection{Deducing anisotropic drained constants from undrained ones for very hetero- geneous porous media}

At this point we have determined a data processing scheme that would provide all the drained constants for a poroelasticity system from measurements of the undrained constants. In the example of the preceding subsection, we needed to broaden the meaning of the undrained set of constants to include the Skempton $A_{i}$ coefficients, which were not needed in earlier parts of the paper. But they could nevertheless be computed from the information found earlier too, since we did show how to compute all the $\beta_{i}$ 's directly, and these coefficients provide just the information that we would need for determining these values from (58).

In realistic data collection situations, especially those involving field data, our previous assumptions concerning the nature and orientations of the constituent grains of the granular porous medium may sometimes - perhaps most times - be too idealized. Nevertheless, it is the case that the equations of poroelasticity never become any more complex than those shown here. What does change however is the interpretation of the directional grain moduli. In the worst case scenario, equation (61) needs to be replaced by an equation of the same form, namely:

$$
\frac{1}{3 K_{i}^{*}}=s_{i 1}^{u}+s_{i 2}^{u}+s_{i 3}^{u}-\beta_{i}(1-B)
$$

Our measurements are exactly as before, but the interpretation of the resulting constant estimator $K_{i}^{*}$ becomes that of an effective medium bulk modulus, i.e., one that is (or at least could be) dependent on the directions $i=1,2,3$ of mesaurement. Effective medium theories for random polycrystals generally assume (see [25]) that the anisotropic grains are perfectly randomly oriented. Of course, this may not be true in practice. But to do a better job of predicting the outcome of experiments in situations where grain orientations are not perfectly random, we need information about these deviations from perfect randomness. In the present context, the information would preferably come in the form of these measured constants $K_{i}^{*}$. Some effort should then be expended in showing how such moduli might arise if the constituents' nature and volume fractions are known. But in our current state 
of knowledge, i.e, in the absence of various required measurements, some information about these constants may be sufficient to permit analysis of other results for experiments of most interest in poroelasicity.

Therefore, to provide some quantitative information about how important the anisotropy, as well as the random orientation of the constituents, might be in a few cases, TABLES 1 and 2 provide some quantitative examples based on results in references [25-27]. TABLES 3 and 4, respectively, provide input data for the types of orthorhombic solids [28], and the results for the Voigt, Reuss, and directional measures of bulk moduli for these particular materials. Note the significant finding that the directional moduli do NOT have to stay within the values set by the Voigt and Reuss estimators. Some additional examples are provided in TABLES 5-9.

\section{Summary and Conclusions}

There have been a great many experiments done on poroelastic systems through the years, and many attempts to measure complete poroelastic data sets. The work summarized here is designed to make this process easier by removing the need (whenever possible) for tedious fitting routines that have often been used to find the pertinent drained constants for the measured fluid-saturated and undrained systems. It is also of some real practical importance to have methods like those discussed here, because of the well-known fact that the presence of the pore-fluid can alter the nature of the points of contact between neighboring grains, and therefore alter the values of the "drained" constants that were sought here - and found via the methods developed for this purpose. Rather different values of the "drained" constants might be found if instead all the fluid is physically drained out of the system, so it is effectively "dry" rather than merely "drained" (i.e., in the sense of pore-fluid having the capability of moving in and out of the boundaries as would happen in the absence of jacketing material). In the case of a fully dry system, the grain-to-grain contacts are known to act very differently than they do when saturated with certain fluids. At the very least, it could be important to check experimentally whether these constants are different or not in a variety of systems, and the present analysis will permit such studies to move forward.

One especially interesting aspect of the analysis is that in no case presented did the solution of any of the problems discussed involve any analysis more complicated that solving 
a linear equation. There are no quadratic equations solved in this paper, and none that needed to be solved. The hardest calculation in the paper is the implicit inversion of a $3 \times 3$ matrix when the real data are poroelastic stiffnesses, rather than compliances. This situation does happen in practice whenever the data are obtained using wave propagation methods. Then, the actual data have the form $v=\sqrt{c / \rho}$, where $v$ is a wave speed, $c$ is a stiffness or combination of stiffnesses, and $\rho$ is the inertial density. A complete set of the stiffnesses for the principal stresses and strains is needed for our analysis because we require the compliance data, and to obtain a complete set of compliance data from stiffness data, we also require a complete set of the corresponding stiffness data. We need all the elements of the undrained $3 \times 3$ compliance matrix for the principal stresses and strains in order to proceed.

The analysis has been restricted to systems having orthotropic poroelastic symmetry or higher. Lower symmetry systems might also be studied, but we purposely avoided them here because for such systems it is harder to know for sure from experimental data when you have found the true axes of symmetry. Also, in these cases of orthotropic symmetry, the system of equations is reduced because there is no coupling of the fluid effects to the shear components associated with the twisting strains $e_{23}, e_{31}, e_{11}$, or the stresses $\sigma_{23}, \sigma_{31}, \sigma_{12}$. Shear effects are not ignored altogether however, as there are well-known shearing mechanisms in poroelastic media associated with Skempton's coefficient $A[19,24]$. These effects were studied here, and were found to be very useful in accomplishing our main goals, since they provided a necessary mechanism for measuring some otherwise difficult to measure off-diagonal terms in the poroelastic equations.

We conclude that this analysis has been successful in solving the problem of obtaining drained constants from undrained constants in all the cases considered so far. The chosen set of cases (orthotropic or higher symmetry) is not very restrictive from a practical point of view, as the great majority of poroelastic systems studied in practice actually have hexagonal (transversely isotropic) symmetry or higher, and therefore are all explicitly included within the range of the present analyses. 


\section{Acknowledgments}

The author thanks Seiji Nakagawa and Leon Thomsen for helpful comments on the work. Work performed under the auspices of the U.S. Department of Energy, at the Lawrence Berkeley National Laboratory under Contract No. DE-AC02-05CH11231. Support was provided specifically by the Geosciences Research Program of the DOE Office of Basic Energy Sciences, Division of Chemical Sciences, Geosciences and Biosciences.

\section{REFERENCES}

[1] F. Gassmann, "Über die Elastizität poröser Medien," Vierteljahrsschrift der Naturforschenden Gesellschaft in Zürich 96 (1951) 1-23.

[2] M. A. Biot, D. G. Willis, The elastic coefficients of the theory of consolidation, Journal of Applied Mechanics 24 (1957) 594-601.

[3] R. J. S. Brown, J. Korringa, On the dependence of the elastic properties of a porous rock on the compressiblity of the pore fluid, Geophysics 40 (1975) 608-616.

[4] J. R. Rice, M. P. Cleary, Some basic stress diffusion solutions for fluid-saturated elastic porous media with compressible constituents, Reviews of Geophysics and Space Physics 14 (1976) 227-241.

[5] L. Thigpen and J. G. Berryman, Mechanics of porous elastic materials containing mutiphase fluid, International Journal of Engineering Science 23 (1985) 1203.

[6] R. W. Zimmerman, Compressibility of Sandstones, Elsevier, Amsterdam, 1991, Chapter 6.

[7] A. H.-D. Cheng, Material coefficients of anisotropic poroelasticity, International Journal of Rock Mechanics 34 (1997) 199-205.

[8] H. F. Wang, Theory of Linear Poroelasticity with Applications to Geomechanics and Hydrogeology, Princeton University Press, Princeton, NJ, 2000.

[9] A. W. Wood, A Textbook of Sound, Bell, London, 1955, p. 360. 
[10] X. Zhu. G. A. McMechan, Direct estimation of the bulk modulus of the frame in a fluid-saturated elastic medium by Biot theory, in 60th International Meeting, Society of Exploration Geophysicists, Expanded Abstracts, pp. 787-790 (1990).

[11] A. Reuss, "Berechung der Fliessgrenze von Mischkristallen," Z. Angew. Math. Mech. 9 (1929) 55 .

[12] W. Voigt, Lehrbuch der Kristallphysik, Teubner, Leipzig, 1928, p. 962.

[13] R. Hill, The elastic behaviour of crystalline aggregate, Proceedings of the Physical Society of London A65 (1952) 349-354.

[14] J. G. Berryman, G. W. Milton, Exact results for generalized Gassmann's equations in composite porous media with two constituents, Geophysics 56 (1991) 1950-1960.

[15] J. G. Berryman and S. R. Pride, Models for computing geomechanical constants of double-porosity materials from the constituents' properties, Journal of Geophysical Research 107 B3 (2002) 2052-1-2052-15.

[16] G. I. Barenblatt and Y. P. Zheltov, Fundamental equations of filtration of homogeneous liquids in fissured rocks, Sov. Phys. Dokl. 132 (1960) 545-548.

[17] J. G. Berryman and H. F. Wang, The elastic coefficients of double-porosity models for fluid transport in jointed rock, Journal of Geophysical Research 100 (1995) 24611-24627.

[18] B. Gurevich, D. Makarynska, M. Pervukhina, Ultrasonic moduli for fluid-saturated rocks: Mavko-Jizba relations rederived and generalized, Geophysics 74 (2009) N25-N30.

[19] A. W. Skempton, The pore-pressure coefficients $A$ and $B$, Géotechnique 4 (1954) 143147.

[20] R. W. Zimmerman, Coupling in poroelasticity and thermoelasticity, International Journal of Rock Mechanics 37 (2000) 79-87.

[21] P. A. Berge and J. G. Berryman, Realizability of negative pore compressibility in poroelastic composites, ASME Journal of Applied Mechanics 62 (1995) 1053-1062. 
[22] J. G. Berryman, Transversely isotropic elasticity and poroelasticity arising from thin isotropic layers, in Theoretical and Computational Acoustics 1997, edited by Y.-C. Teng and E.-C. Shang and Y.-H. Pao and M. H. Schultz and A. D. Pierce, World Scientific, Newark, NJ, 1997, pp. 457-474.

[23] R. D. Stoll, Acoustic waves in saturated sediments, in Physics of Sound in Marine Sediments, edited by L. Hampton, Plenum, New York, 1974, pp. 19-39.

[24] D. A. Lockner and S. A. Stanchits, Undrained poroelastic response of sandstones to deviatoric stress change, Journal of Geophysical Research 107 (2002) 2353-1-2353-14.

[25] J. G. Berryman, Bounds and self-consistent estimates for elastic constants of random polycrystals with hexagonal, trigonal, and tetragonal symmetries, Journal of the Mechanics and Physics of Solids 53 (2005) 2141-2173.

[26] S. I. Ranganathan and M. Ostoja-Starzewski, Universal elastic anisotropy index, Physical Review Letters 101 (2008) 055502.

[27] S. I. Ranganathan and M. Ostoja-Starzewski, Mesoscale conducticity and scaling function in aggregates of cubic, hexagonal, and tetragonal crystals, Physical Review B 77 (2008) 214308.

[28] M. J. P. Musgrave, Crystal Acoustics: Introduction to the Study of Elastic Waves and Vibrations in Crystals, Acoustical Society of America, New York, 2003, pp. 278-281.

[29] G. Simmons and H. Wang, Crystal Elastic Constants and Calculated Aggregate Properties: A Handbook, The M.I.T. Press, Cambridge, Massachusetts, 1971. 
TABLE 1. Reuss (R), Voigt (V), and self-consistent effective (*) bulk moduli of various common anisotropic materials [25]: Water ice, cadmium, zinc, graphite, $\alpha$-quartz, corundum, barium titanate, rutile, aluminum, copper, magnesia, spinel. Full references for the data used in both TABLES 1 and 2 are provided in reference [25]. Units of bulk modulus $K$ are GPa.

\begin{tabular}{|c|c|c|c|c|c|}
\hline \hline Material & Symmetry & $K_{R}$ & $K^{*}$ & $K_{V}$ & $K_{V} / K_{R}$ \\
\hline $\mathrm{H}_{2} \mathrm{O}$ & Hexagonal & 8.89 & 8.89 & 8.89 & 1.00 \\
$\mathrm{Cd}$ & Hexagonal & 48.8 & 54.7 & 58.1 & 1.19 \\
$\mathrm{Zn}$ & Hexagonal & 61.6 & 70.9 & 75.1 & 1.22 \\
$\mathrm{Graphite}$ & Hexagonal & 35.8 & 88.0 & 286.3 & 8.00 \\
\hline $\mathrm{Al}_{2} \mathrm{O}_{3}$ & Trigonal & 253.5 & 253.7 & 253.9 & 1.002 \\
$\alpha-\mathrm{SiO}_{2}$ & Trigonal & 37.6 & 37.8 & 38.1 & 1.01 \\
\hline $\mathrm{TiO}_{2}$ & Tetragonal & 209 & 213 & 218 & 1.04 \\
$\mathrm{BaTiO}_{2}$ & Tetragonal & 163.1 & 179.3 & 186.8 & 1.15 \\
\hline $\mathrm{Al}_{\mathrm{MgO}}$ & Cubic & 76.3 & 76.3 & 76.3 & 1.00 \\
$\mathrm{MgAl}_{2} \mathrm{O}_{4}$ & Cubic & 162.4 & 162.4 & 162.4 & 1.00 \\
$\mathrm{Cu}^{2}$ & Cubic & 138.0 & 138.0 & 138.0 & 1.00 \\
\hline \hline
\end{tabular}


TABLE 2. Reuss (R), Voigt (V), and self-consistent effective $(*)$ shear moduli of various common materials [25]: Water ice, cadmium, zinc, graphite, $\alpha$-quartz, corundum, barium titanate, rutile, aluminum, copper, magnesia, and spinel. Units of shear modulus $G$ are GPa. The anisotropy parameter $\mathcal{A} \equiv 5 \frac{G_{V}}{G_{R}}+\frac{K_{V}}{K_{R}}-6$ (from $[26,27]$ ).

\begin{tabular}{|c|c|c|c|c|c|c|}
\hline \hline Material & Symmetry & $G_{R}$ & $G^{*}$ & $G_{V}$ & $G_{V} / G_{R}$ & $\mathcal{A}$ \\
\hline $\mathrm{H}_{2} \mathrm{O}$ & Hexagonal & 3.48 & 3.52 & 3.55 & 1.02 & 0.10 \\
$\mathrm{Cd}$ & Hexagonal & 22.1 & 24.3 & 26.4 & 1.19 & 1.14 \\
$\mathrm{Zn}$ & Hexagonal & 34.1 & 40.6 & 44.8 & 1.31 & 1.77 \\
Graphite & Hexagonal & 9.2 & 52.6 & 219.4 & 23.8 & 121.0 \\
\hline $\mathrm{Al}_{2} \mathrm{O}_{3}$ & Trigonal & 160.7 & 163.1 & 165.5 & 1.03 & 0.15 \\
$\alpha-\mathrm{SiO}_{2}$ & Trigonal & 41.0 & 44.0 & 47.6 & 1.16 & 0.81 \\
\hline $\mathrm{TiO}_{2}$ & Tetragonal & 99.5 & 114.5 & 124.9 & 1.26 & 1.34 \\
$\mathrm{BaTiO}_{2}$ & Tetragonal & 47.4 & 53.6 & 59.8 & 1.26 & 1.46 \\
\hline $\mathrm{Al}_{\mathrm{MgO}}$ & Cubic & 26.0 & 26.2 & 26.3 & 1.01 & 0.05 \\
$\mathrm{MgAl}_{2} \mathrm{O}_{4}$ & Cubic & 123.9 & 126.3 & 128.6 & 1.04 & 0.20 \\
$\mathrm{Cu}^{2}$ & Cubic & 40.0 & 46.3 & 51.3 & 1.28 & 1.41 \\
\hline \hline
\end{tabular}


TABLE 3. Data for the principal stiffness coefficients $c_{i j}$ of orthorhombic sulfur $(\mathrm{S})$, Rochelle salt, Benzophenone, and $\alpha$-Uranium ( $\alpha$-U). All data from Musgrave [28], but re-expressed in units of GPa.

\begin{tabular}{|c|c|c|c|c|}
\hline \hline Stiffness & Sulfur (S) & Rochelle Salt & Benzophenone & $\alpha$-Uranium \\
\hline$c_{11}$ & 24.0 & 25.5 & 107.0 & 215.0 \\
$c_{22}$ & 20.5 & 38.1 & 100.0 & 199.0 \\
$c_{33}$ & 48.3 & 37.1 & 71.0 & 267.0 \\
$c_{12}$ & 13.3 & 14.1 & 55.0 & 46.0 \\
$c_{13}$ & 17.1 & 11.6 & 16.9 & 22.0 \\
$c_{23}$ & 15.9 & 14.6 & 32.1 & 107.0 \\
\hline \hline
\end{tabular}


TABLE 4. Data for various measures of bulk modulus $K$ (Voigt, Reuss, and three partial sum moduli) for orthorhombic sulfur (S), Rochelle salt, Benzophenone, and $\alpha$-Uranium $(\alpha-\mathrm{U})$. All data from Musgrave [28] (see TABLE 3 here), while the expressions in the main text were used for the computations. All moduli in units of GPa.

\begin{tabular}{|c|c|c|c|c|}
\hline \hline Bulk Modulus & Sulfur (S) & Rochelle Salt & Benzophenone & $\alpha$-Uranium \\
\hline$K_{V}$ & 20.6 & 20.1 & 54.0 & 114.6 \\
$K_{R}$ & 17.6 & 19.3 & 49.2 & 111.3 \\
$K_{1}$ & 15.2 & 12.5 & 55.8 & 87.9 \\
$K_{2}$ & 10.1 & 30.6 & 107.5 & 113.6 \\
$K_{3}$ & 15.8 & 23.3 & 29.6 & 147.7 \\
\hline \hline
\end{tabular}


TABLE 5. Data for the principal stiffness coefficients $c_{i j}$ for $i, j=1,2,3$, as well as $c_{44}$, of hexagonal minerals: cadmium $(\mathrm{Cd}), \mathrm{H}_{2} \mathrm{O}$ ice, $\beta$-quartz $\left(\mathrm{SiO}_{2}\right)$, titanium $(\mathrm{Ti})$, and zirconium (Zr). All data from Simmons and Wang [29] (entry numbers: 52473, 52563, 52643, 52726, and 52798, respectively), but re-expressed in units of GPa.

\begin{tabular}{|c|c|c|c|c|c|}
\hline \hline Stiffness & Cadmium (Cd) & $\mathrm{H}_{2}$ O Ice & $\beta$-Quartz & Titanium (Ti) & Zirconium (Zr) \\
\hline$c_{11}$ & 115.30 & 13.85 & 116.6 & 163.9 & 137.0 \\
$c_{33}$ & 51.20 & 14.99 & 110.4 & 181.6 & 160.7 \\
$c_{12}$ & 39.24 & 7.07 & 16.7 & 91.3 & 75.6 \\
$c_{13}$ & 40.22 & 5.81 & 32.8 & 68.9 & 65.4 \\
$c_{44}$ & 20.40 & 3.19 & 36.1 & 47.2 & 30.1 \\
\hline \hline
\end{tabular}


TABLE 6. Data for various measures of bulk modulus $K$ (Voigt, Reuss, and three partial-sum moduli) for hexagonal minerals: cadmium $(\mathrm{Cd}), \mathrm{H}_{2} \mathrm{O}$ ice, $\beta$-quartz $\left(\mathrm{SiO}_{2}\right)$, titanium (Ti), and zirconium (Zr). All data from Simmons and Wang [29] (see TABLE 5), while the expressions in the main text were used for all the computations. All moduli in units of GPa.

\begin{tabular}{|c|c|c|c|c|c|}
\hline \hline Bulk Modulus & Cadmium $(\mathrm{Cd})$ & $\mathrm{H}_{2}$ O Ice & $\beta$-Quartz & Titanium (Ti) & Zirconium (Zr) \\
\hline$K_{V}$ & 57.89 & 8.90 & 56.47 & 107.51 & 94.17 \\
$K_{R}$ & 48.61 & 8.90 & 56.37 & 107.50 & 94.02 \\
$K_{1}=K_{2}$ & 143.07 & 8.94 & 53.97 & 109.00 & 89.58 \\
$K_{3}$ & 20.95 & 8.82 & 61.86 & 104.63 & 104.36 \\
\hline \hline
\end{tabular}


TABLE 7. Data for the principal stiffness coefficients $c_{i j}$ for $i, j=1,2,3$ and $c_{44}$ of cubic symmetry minerals: aluminum $(\mathrm{Al})$, copper $(\mathrm{Cu})$, magnesia $(\mathrm{MgO})$, and spinel $\left(\mathrm{MgAl}_{2} \mathrm{O}_{4}\right)$. All data from Simmons and Wang [29] (entry numbers: 10089, 10385, 10902, and 11877, respectively), but re-expressed in units of GPa.

\begin{tabular}{|c|c|c|c|c|}
\hline \hline Stiffness & Aluminum $(\mathrm{Al})$ & Copper $(\mathrm{Cu})$ & Magnesia $(\mathrm{MgO})$ & Spinel $\left(\mathrm{MgAl}_{2} \mathrm{O}_{4}\right)$ \\
\hline$c_{11}$ & 107.30 & 170.98 & 297.08 & 298.57 \\
$c_{12}$ & 60.80 & 123.99 & 95.36 & 153.72 \\
$c_{44}$ & 28.30 & 75.45 & 156.13 & 157.58 \\
\hline \hline
\end{tabular}


TABLE 8. Data for various measures of bulk modulus $K$ (Voigt, Reuss, and three partial-sum moduli) for cubic symmetry minerals: aluminum $(\mathrm{Al})$, copper $(\mathrm{Cu})$, magnesia $(\mathrm{MgO})$, and spinel $\left(\mathrm{MgAl}_{2} \mathrm{O}_{4}\right)$. All data from Simmons and Wang [29] (see TABLE 7), while the expressions in the main text were used for all the computations. All moduli in units of GPa. Clearly, all the pertinent bulk moduli for each material are the same (i.e., $K_{V}=K_{R}=K_{1}=K_{2}=K_{3}$, even though these cubic symmetry minerals are not isotropic.

\begin{tabular}{|l|c|c|c|c|}
\hline \hline Bulk Modulus & Aluminum $(\mathrm{Al})$ & Copper $(\mathrm{Cu})$ & Magnesia $(\mathrm{MgO})$ & Spinel $\left(\mathrm{MgAl}_{2} \mathrm{O}_{4}\right)$ \\
\hline$K_{V}=K_{R}=\ldots$ & 76.3 & 139.65 & 162.6 & 202.00 \\
\hline \hline
\end{tabular}


TABle 9. Reuss (R) and Voigt (V) shear moduli of various common hexagonal and cubic materials [29]: cadmium, $\beta$-quartz, titanium, zirconium, aluminum, copper, magnesia, and spinel. Units of shear modulus $G$ are GPa. All the formulas needed to compute the various effective moduli from the stiffness coefficients are given in reference [25]. The anisotropy parameter $\mathcal{A} \equiv 5 \frac{G_{V}}{G_{R}}+\frac{K_{V}}{K_{R}}-6$ (from $\left.[26,27]\right)$.

\begin{tabular}{|c|c|c|c|c|c|}
\hline \hline Material & Symmetry & $G_{R}$ & $G_{V}$ & $G_{V} / G_{R}$ & $\mathcal{A}$ \\
\hline $\mathrm{Cd}$ & Hexagonal & 22.1 & 26.4 & 1.197 & 1.174 \\
$\mathrm{SiO}_{2}$ & Hexagonal & 3.48 & 3.55 & 1.025 & 0.125 \\
$\mathrm{Ti}$ & Hexagonal & 34.1 & 44.8 & 1.31 & 0.154 \\
$\mathrm{Zr}$ & Hexagonal & 32.54 & 33.40 & 1.03 & 0.132 \\
\hline $\mathrm{Al}$ & Cubic & 26.04 & 26.28 & 1.009 & 0.045 \\
$\mathrm{Cu}$ & Cubic & 40.04 & 54.67 & 1.365 & 1.825 \\
$\mathrm{MgO}_{\mathrm{MgAl}} \mathrm{O}_{4}$ & Cubic & 128.06 & 134.02 & 1.047 & 0.235 \\
\hline \hline
\end{tabular}

